\title{
Classifying Disease in Fruit using Machine Learning
}

\author{
Ayeesha ${ }^{*}$, Fathima Zeela ${ }^{2}$, Vijetha ${ }^{3}$ \\ Computer Science and Engineering, Srinivas Institute of Technology, Mangalore, Karnataka, India
}

\section{Article Info \\ Volume 7, Issue 4 \\ Page Number : 309-313}

Publication Issue :

July-August-2021

\section{Article History}

Accepted : 15 July 2021

Published : 22 July 2021

\section{ABSTRACT}

India is agricultural country and Indian farmer select wide selection of fruit and vegetable crops. The cultivation of crops can be improved by the technological support. Fruits and vegetables losses are caused by disease. Diseases are seen on the leaves and fruits of plant, therefore disease detection plays a crucial role in cultivation of crops. Pathogens, fungi, microorganism, bacteria and viruses are sorts of fruit diseases also unhealthy environment is responsible for diseases. There are many techniques to spot diseases in fruits in its early stages. Hence, there's a requirement of automatic fruit unwellness detection system within the early stage of the unwellness. The aim is to detect the fruit disease, this method take input as image of fruit and determine it as infected or non- infected. The proposed method is based on the use of Scale-invariant Feature Transform (SIFT) Model with the desirable goal of accurate and fast classification of fruits. The SIFT features are local and based on the appearance of the object at particular interest points, and are invariant to image scale and rotation. They are also robust to changes in illumination, noise, and minor changes in viewpoint on image processing theory. SIFT have significant advantages because of their high accuracy, relatively easy to extract and allow for correct object identification with low probability of mismatch. Besides, they do not need an outsized number of coaching samples to avoid overfitting.

Keywords : Fruit Diseases, SIFT, python, flask.

\section{INTRODUCTION}

The emerging of new technologies such as digital image processing and image analysis technology has many applications in the biological field. In India, around $78 \%$ of the farmers are marginal and hence they are poor in resources. Therefore, they're not during a position to use the available resources for increasing productivity. A user- friendly software will help the farmer to someextent to detect whether the fruit is diseased or not. The image is processed using the Image Processing techniques and therefore the disease is detected. The disease is detected by our image processing software that helps the farmers to take some precautions. This proves benefits in monitoring large orchards of fruits, and thus automatically detects the diseases as soon as they seem on fruits. The largest sector in the Indian economy is agriculture and it is also the largest employer. So, the agricultural industry places a big role in increasing the Indian economy. Daily products in India are cheaper as compared to other 
parts of the planet for instance 1] In Europe $1 \mathrm{~kg}$ of milk costs $€ 3.54$ (as in Jan. 2021), around 313 rupees, whereas an equivalent quantity of milk costs 46 rupees in India, which is 6.8 times lesser. 2] In Western Europe $1 \mathrm{~kg}$ of apple costs $€ 2.5$ (as in Jan. 2021), around 220 rupees, whereas an equivalent quantity of apple costs 100rs in India. So, this is the average price variation between Milk, Fruits, Vegetables and many other agricultural products like Rice, Wheat compared to other countries. Hence there's tons of scope for export, which may be a new way for the farmer to earn extra money which in term will increase the agricultural income which in turn increase the Indian economy. This is possible only if we produce more fresh fruits. So, this paper focuses on disease detection in fruits which helps in increasing the fresh fruits produced. Fruits like Apple, Grapes and Mango are called cash crops, hence improvement in productivity of those sorts of fruits is required. Various diseases may attack apple fruits mainly Flyspeck, Apple Rot, Sooty Blotch etc. So, its early detection is extremely essential The proposed work mainly gives a review that what steps are performed throughout the entire process to detect particular disease from the fruit. The work proposes a picture processing to affect the most problems with phytopathology that's disease detection and classification. The fruits affected by various diseases caused by fungus, bacteria and the climatic conditions. These diseases are like Bacterial, Parasitic, Fungal, Viral and Fresh fruit. The system uses some images for training, some for-testing purpose then on. The colour images are pre-processed and undergo Euclidian segmentation. The measurements obtained from the study of textural features are given as input to the SIFT Model classifier for training in order to classify it. Finally, system will detect disease of the fruits and will display the output.

\section{RELATED WORK}

A previous research paper presents a review on methods that use digital image processing techniques to detect, recognize and classify plant diseases from digital image and concludes with discussion of more useful problems within the domain and future direction. Another paper discusses the event of portable fruit sorting and grading machine supported computer vision for little agro-industries. The system is meant from low cost material within the sort of inclined and segmented plane to substitute the use of conveyer belt. Another research-oriented paper presents an effective and user-friendly color mapping concept for automated color grading that is well suited for commercial production. User friendliness is usually viewed by the industry as a really important factor to the acceptance and success of automation equipment. Latter work reports on the development of an automatic adjustable algorithm for segmentation of color images, using linear support vector machine (SVM) and Otsu's thresholding method, for apple sorting and grading. The method automatically adjusts the classification hyper plane calculated by using linear SVM and requires minimum training and time. Another paper presents a computer vision based system for automatic grading and sorting of agricultural products like Mango (Mangifera indica L.) based on maturity level. The application of machine vision based system, aimed to exchange manual based technique for grading and sorting of fruit.

\section{SYSTEM IMPLEMENTATION}

The proposed system is implemented in the following architecture. The system takes a fruit image as input. The input is validated in order to maintain data integrity. Once validation is completed the SIFT technique is implemented where the entire process is divided into 4 phases. At first phase Constructing a 
Scale Space is established, to make sure that features are scale-independent. At second phase Key point Localisation is done in order to identify the suitable features or key points. Later on at phase three Orientation Assignment is initiated in order to ensure the key points are rotation invariant. At the final phase Key point Descriptor is constructed to assign a unique fingerprint to each key point. Overall technique includes feature extraction from input images. Once the sparse matrix is created after this process the output is passed to trained model which matches the sparse matrix with the pretrained images and predicts the disease name. The GUI is designed using flask module with HTML,CSS and backend is programmed with python script.

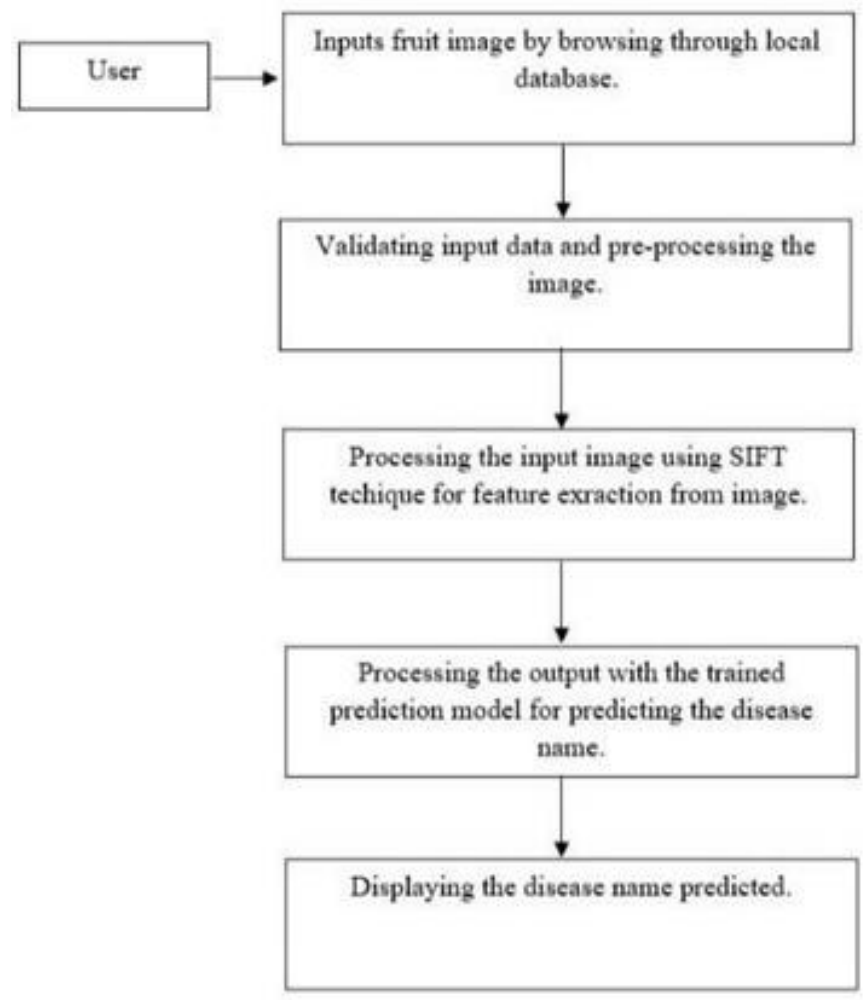

Figure 1. System Design of Proposed Methodology

The data flow design of the proposed system is designed in the flowing way the input data image is converted into a grey scale format and key points from the images are recorded and a matrix is created using SIFT feature extraction technique. In the below figure we can see that input is pre- processed and verification of data is done in order to obtain robustness in proposed system. The pre trained is obtained by following the similar data flow scenario which is dataset images, feature extractions and storing in model of training. The dataset images consist of images from internet on various fruit diseases classifies into four main categories which are bacterial, fungal, parasitic and viral. The model predicts the disease classification based on the input dataset trained.

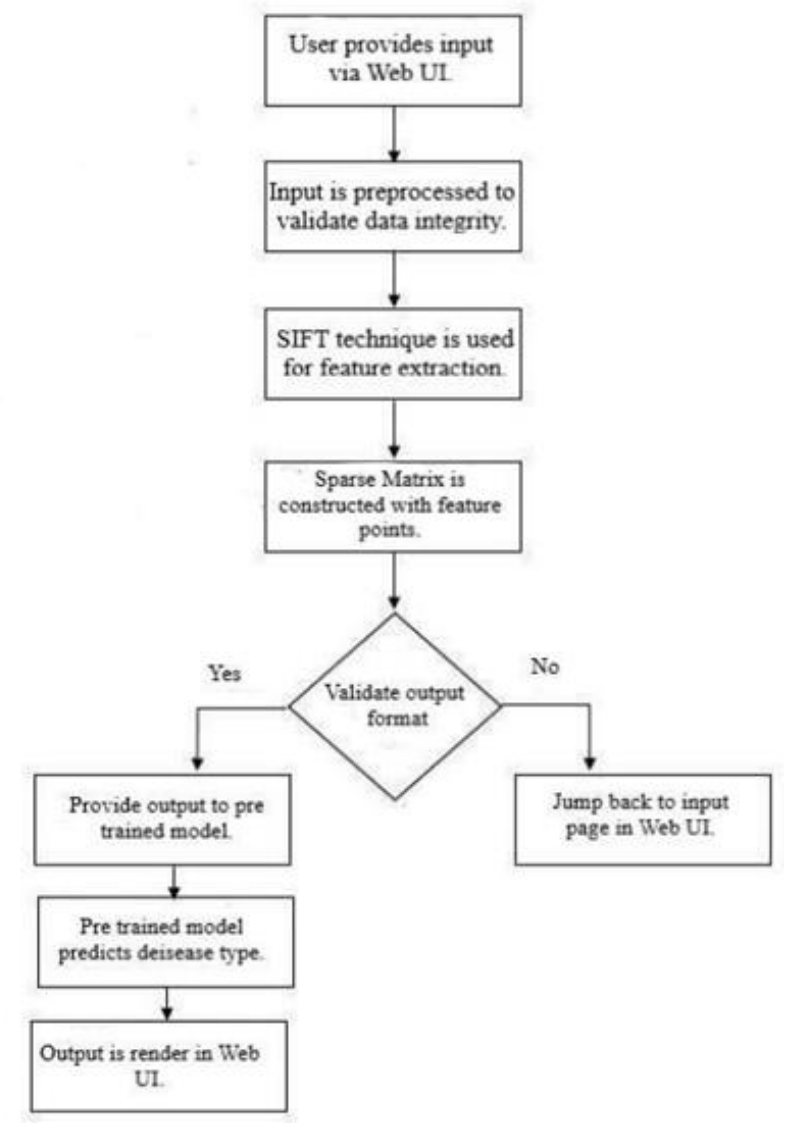

Figure 2. Data Flow design of Proposed System

\section{EXPERIMENTAL RESULT}

The system is being designed and following outputs have been obtained as results.

In figure 3 shows the user options to login or create a account in order to access the proposed system portal 


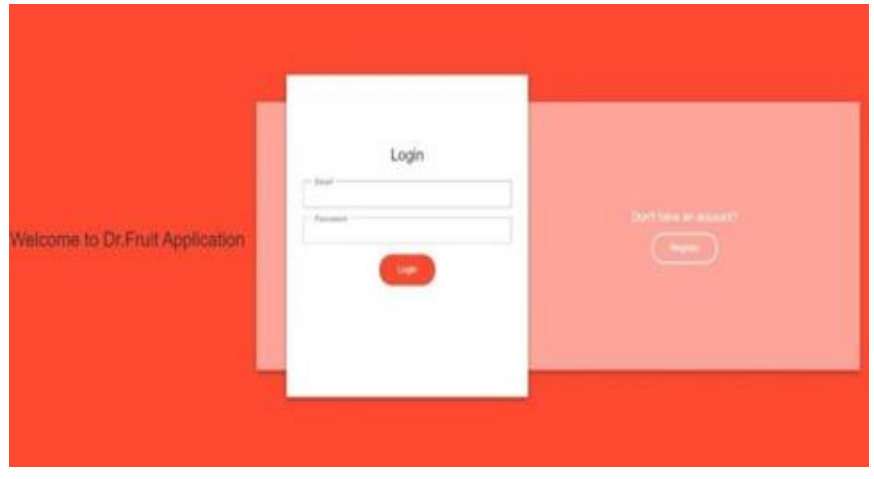

Figure 3. Login/Signup Page

In figure 4 shows the Home page consisting of description of proposed system features and user manual.

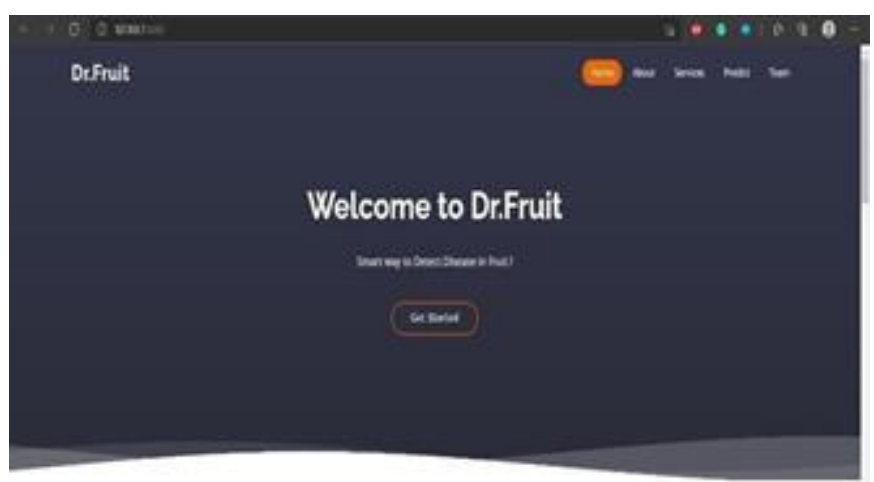

Figure 4. Home page

In figure 5 shows the prediction page where user can upload the input image and analyse the disease in fruits.
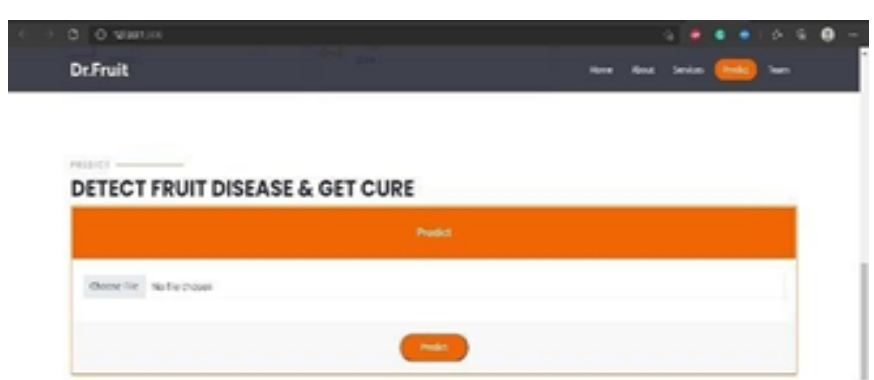

?

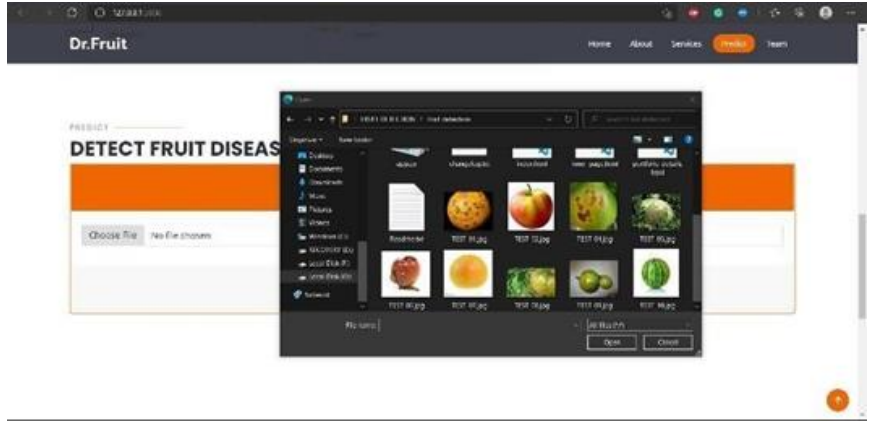

Figure 6. Input Selection Page

In figure 7 shows the final prediction result obtained for user input

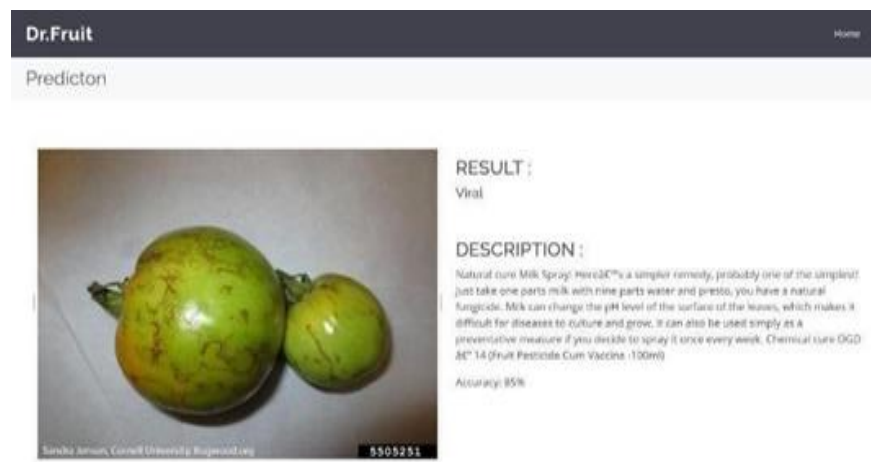

Figure 7. Output page

\section{CONCLUSION}

An Machine learning based solution is proposed and evaluated in this paper for the detection and classification of fruit diseases. This paper presents the innovative methods to detect diseases in fruits and is also a prototype in implementation of the usage of modern technology in agriculture, especially in cash crops. The disease is detected in a very cheap and fast wayIt would also promote Indian Farmers to do smart farming which helps to take time to time decisions which also save time and reduce loss of fruit due to diseases. The leading objective of our paper is to enhance the value of fruit disease detection.

Figure 5. Prediction Page. 


\section{REFERENCES}

[1]. Hongjun wang, Qisong Mou, Youjun Yue, Hui Zhao: "Research on Detection Technology of various Fruit Disease Spots Based on Mask RCNN", Tianjin University of Technology, Tianjin China, 2016

[2]. Kulkarni Anand H, Ashwin Patil RK: "Applying image processing technique to detect plant diseases". Int J Mod Eng Res, 2012.

[3]. S .B. Ullagaddi and S. Viswanadha Raju: "Disease Recognition in Mango Crop Using Modified Rotational Kernel Transform Features". 2017 Internationals.

\section{Cite this article as :}

Ayeesha, Fathima Zeela, Vijetha, "Classifying Disease in Fruit using Machine Learning", International Journal of Scientific Research in Computer Science, Engineering and Information Technology (IJSRCSEIT), ISSN : 2456-3307, Volume 7 Issue 4, pp. 309-313, July-August 2021. Available at doi : https://doi.org/10.32628/CSEIT217341 Journal URL : https://ijsrcseit.com/CSEIT217341 